\title{
Chiari-related hydrocephalus: assessment of clinical risk factors in a cohort of 297 consecutive patients
}

\author{
Jian Guan, MD, ${ }^{1}$ Jay Riva-Cambrin, MD, MSc, ${ }^{2}$ and Douglas L. Brockmeyer, MD1 \\ 'Division of Pediatric Neurosurgery, Department of Neurosurgery, Primary Children's Hospital, University of Utah, Salt Lake City, \\ Utah; and 'Department of Clinical Neurosciences, Foothills Medical Centre, University of Calgary, Alberta, Canada
}

\begin{abstract}
OBJECTIVE Patients treated for Chiari I malformation (CM-I) with posterior fossa decompression (PFD) may occasionally and unpredictably develop postoperative hydrocephalus. The clinical risk factors predictive of this type of Chiari-related hydrocephalus $(\mathrm{CRH})$ are unknown. The authors' objective was to evaluate their experience to identify risk factors that may predict which of these patients undergoing PFD will develop $\mathrm{CRH}$ after surgery.

METHODS The authors performed a retrospective clinical chart review of all patients who underwent PFD surgery and duraplasty for CM-I at the Primary Children's Hospital in Utah from June 1, 2005, through May 31, 2015. Patients were dichotomized based on the need for long-term CSF diversion after PFD. Analysis included both univariate and multivariable logistic regression analyses.

RESULTS The authors identified 297 decompressive surgeries over the period of the study, 22 of which required longterm postoperative CSF diversion. On multivariable analysis, age $<6$ years old (OR 3.342, 95\% Cl 1.282-8.713), higher intraoperative blood loss (OR 1.003, 95\% Cl 1.001-1.006), and the presence of a fourth ventricular web (OR 3.752, 95\% $\mathrm{Cl}$ 1.306-10.783) were significantly associated with the need for long-term CSF diversion after decompressive surgery.

CONCLUSIONS Younger patients, those with extensive intraoperative blood loss, and those found during surgery to have a fourth ventricular web were at higher risk for the development of $\mathrm{CRH}$. Clinicians should be alert to evidence of $\mathrm{CRH}$ in this patient population after PFD surgery.
\end{abstract}

http://thejns.org/doi/abs/10.3171/2016.8.FOCUS16203

KEY WORDS Chiari malformation; hydrocephalus; ventriculoperitoneal shunt; posterior fossa decompression

$\mathrm{C}$ HIARI I malformation (CM-I), defined as herniation of the cerebellar tonsils $>5 \mathrm{~mm}$ below the foramen magnum, ${ }^{11,13}$ is seen radiographically in up to $4 \%$ of the pediatric population. ${ }^{14}$ The most common treatment modality for patients with symptomatic CM-I is posterior fossa decompression (PFD), either with or without duraplasty. ${ }^{3,59}$ Although decompression alone is frequently effective and is associated with a low complication rate, ${ }^{12}$ some patients require further interventions during or after the initial decompression. These may include additional decompressions because of persistent symptoms/recurrent herniation,,$^{10}$ implantation of syrinx shunting systems for syringomyelia, ${ }^{2}$ and occipitocervical fusion because of instability. ${ }^{4}$

Hydrocephalus may be seen in association with CM$\mathrm{I}^{13}$ likely because of disruptions in normal CSF flow. ${ }^{6}$ Although patients sometimes demonstrate evidence of hy- drocephalus during their initial presentation for CM-I, a subset of patients appear to develop hydrocephalus only after decompressive surgery. ${ }^{15}$ These patients may present with evidence of raised intracranial pressure, ventricular dilation on imaging, or persistent CSF leak postoperatively. To date, there are no reports in the literature investigating what factors are associated with the need for CSF diversion after PFD is performed to treat CM-I. In our study, we investigated the clinical factors associated with the development of Chiari-related hydrocephalus (CRH) necessitating long-term CSF diversion.

\section{Methods \\ Data Collection}

Institutional review board approval from the University of Utah Medical Center and Primary Children's Medical

ABBREVIATIONS CM-I = Chiari I malformation; $\mathrm{CRH}=$ Chiari-related hydrocephalus; $\mathrm{PFD}=$ posterior fossa decompression.

SUBMITTED May 5, 2016. ACCEPTED August 8, 2016.

INCLUDE WHEN CITING DOI: 10.3171/2016.8.FOCUS16203. 
Center was obtained for all study-related activities. We performed a retrospective chart review using a pediatric neurosurgical operative database to identify all patients who underwent PFD and duraplasty between June 1, 2005, and May 31, 2015, at Primary Children's Medical Center.

Demographic information on all patients was collected and included sex, age at time of decompression surgery, ethnicity, insurance type (private vs Medicaid), and number of prior PFD surgeries. Intraoperative variables collected included the need for concurrent or subsequent occipitocervical fusion, intraoperative blood loss, and surgery type (duraplasty alone, duraplasty with tonsillar shrinking, or duraplasty with tonsillar resection). Each operative report was also scrutinized for the presence of a "web," "veil," or "arachnoid adhesion" over the fourth ventricular outlet during surgery. A fourth ventricular web is a layer of tissue that covers and disrupts the flow of CSF from the fourth ventricle and requires dissection. Postoperative variables including the development of a CSF leak, wound complications (i.e., pseudomeningocele, superficial infection, or deep infection), non-wound-related complications (e.g., chemical meningitis requiring readmission, pneumonia), and length of hospital stay were also recorded.

Patients were dichotomized into hydrocephalus and nohydrocephalus groups based on strict criteria. Those in the hydrocephalus group must have undergone a ventricular shunt placement/endoscopic third ventriculostomy procedure within 12 months of their decompressive surgery and could not have had a prior CSF diversion procedure. For patients who had multiple PFD surgeries during the study period, each procedure was recorded as a separate case. If these patients required CSF diversion, only the decompression immediately prior to the CSF diversion procedure was counted in the hydrocephalus group.

\section{Surgical Technique}

All surgeries were performed by one of 5 pediatric neurosurgeons at a single institution. The precise procedure with regard to the extent of decompression and the performance of duraplasty/tonsillar shrinking/tonsillar resection was based on preoperative and intraoperative findings specific to each patient. Each patient underwent bony decompression of the craniocervical junction, including wide opening of the posterolateral foramen magnum and a C-1 laminectomy. The dura was then opened in a Yshaped fashion, and the fourth ventricle was explored. In the majority of patients, the cerebellar tonsils were shrunk using bipolar cautery; in extreme cases, tonsillar resection was performed. The fourth ventricular outlet was explored whenever possible to confirm CSF flow. During the exploration, any obstructive web/veil was noted and cleared. The dura was closed using dural substitute (Durepair, Medtronic Corp.) in a watertight fashion.

Postoperatively, pain was controlled using a combination of nonsteroidal antiinflammatory drugs, opioids, and muscle relaxants. Patients were encouraged to mobilize and were discharged when they could tolerate food by mouth and could walk around the hospital unassisted.

\section{Statistical Analysis}

After dichotomization of the patients into the hydrocephalus or no-hydrocephalus group as described above, we conducted both univariate analysis and multivariable logistic regression analysis. For demographic variables, age was dichotomized as $<6$ years and $\geq 6$ years. This age was selected based on the distribution of shunt placement timing in our patients (Fig. 1) and a previously published report. ${ }^{1}$ Intraoperative blood loss values were not available for 42 patients (14.1\%), and for these patients the median blood loss of all patients $(30 \mathrm{ml})$ was substituted for multivariable analysis. These substitutions did not have a significant impact on mean intraoperative blood loss for the two groups (mean blood loss with substitutions of $56.6 \pm 90.9$ $\mathrm{ml}$ for the no-hydrocephalus group vs $61.2 \pm 97.8 \mathrm{ml}$ without substitutions, $\mathrm{p}=0.579$; mean blood loss with substitu-

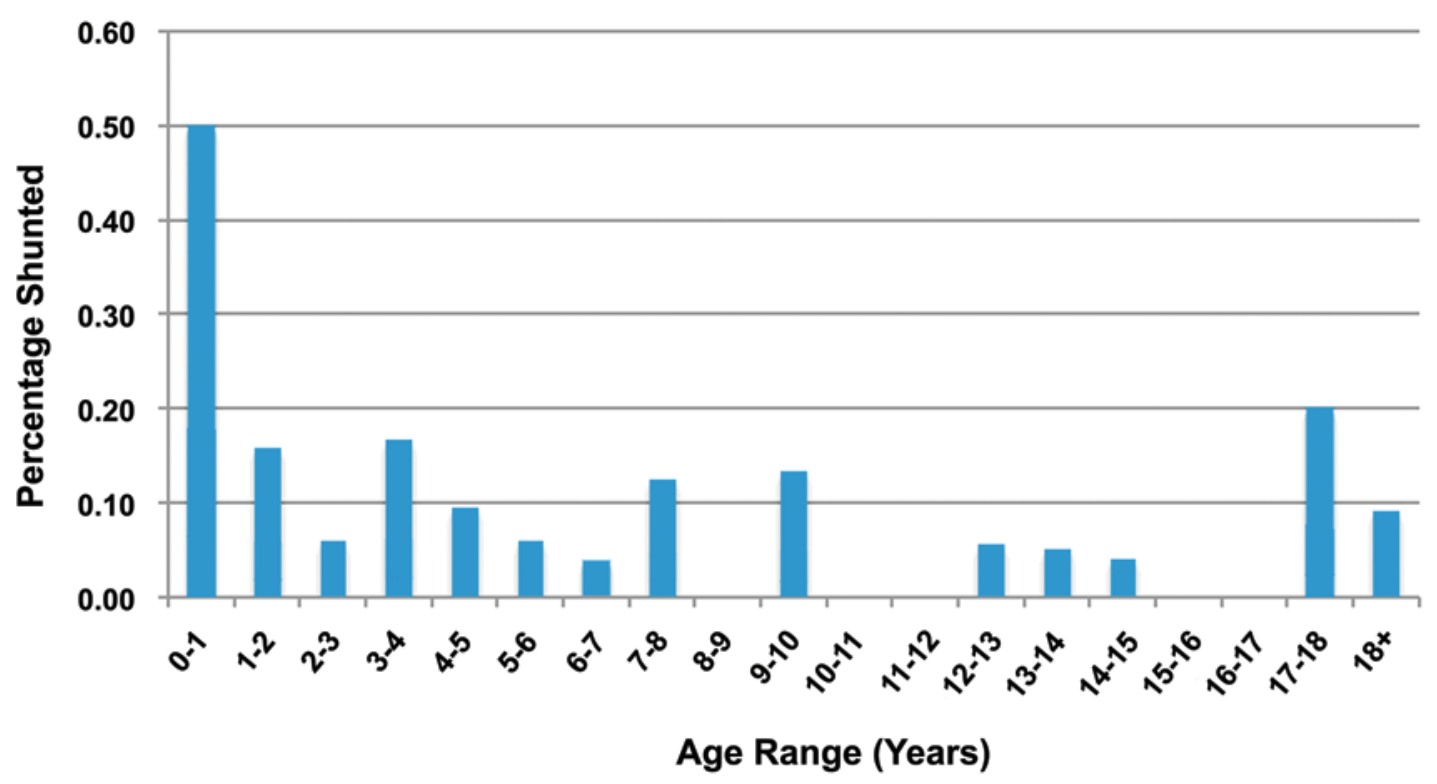

FIG. 1. Percentage of patients at each age who underwent shunt implantation. 
tions of $126.6 \pm 187.6 \mathrm{ml}$ for the hydrocephalus group vs $131.2 \pm 190.9 \mathrm{ml}$ without substitutions, $\mathrm{p}=0.937$ ).

In our univariate analysis, the chi-square test was used to compare categorical variables, while t-tests were used to evaluate continuous variables. For our multivariable logistic regression, we initially included all factors with a $\mathrm{p}$ $<0.05$ on univariate analysis. Complications, CSF leak, fusion, and length of stay were removed from the model because we thought it possible that these may have been directly associated with the outcome of interest (need for CSF diversion). Statistical significance in all analyses was defined as $\mathrm{p}<0.05$.

All statistical analysis was performed using SPSS V20.0 (IBM Corporation).

\section{Results}

Over the study period, 283 patients underwent a total of 297 PFD procedures for CM-I. Of these procedures, 22 required a subsequent CSF diversion procedure within the subsequent 12 months (Table 1), with 21 patients receiving a ventricular shunt and 1 patient undergoing an endoscopic third ventriculostomy. Reasons for CSF diversion included increasing ventricular size (14 patients), persistent CSF leak/pseudomeningocele (4 patients), persistent/ worsening of cerebellar tonsil herniation (3 patients), and uncontrollable headache during postoperative external ventricular drain weaning (1 patient).

Demographically, patients in the hydrocephalus and no-hydrocephalus groups were similar in racial/ethnic background $(p=0.354)$, sex distribution $(p=0.780)$, and insurance profiles $(\mathrm{p}=0.150)$, but a larger percentage of patients in the hydrocephalus group were in the younger age group $(59.1 \%$ vs $31.3 \%, \mathrm{p}=0.008)$. Patients requiring CSF diversion were more likely to have had a previous decompression for CM-I $(\mathrm{p}<0.001)$ and to require a fusion during or after the decompression $(31.8 \%$ vs $10.5 \%$, $\mathrm{p}=$ $0.003)$. Although the two groups were statistically similar in the type of surgery performed $(p=0.062)$, patients in the hydrocephalus group were far more likely to have a fourth ventricular web discovered intraoperatively (40.9\% vs $13.5 \%, p=0.001$ ) and to have had higher mean intraoperative blood loss $(126.6 \pm 187.6 \mathrm{ml}$ vs $56.6 \pm 90.9 \mathrm{ml}$, $\mathrm{p}=0.002$ ). Postoperatively, patients in the hydrocephalus group were more likely to experience a CSF leak $(22.7 \%$ vs $4.4 \%, p<0.001)$ or wound complications $(\mathrm{p}<0.001)$. These patients were also more likely to suffer from nonwound-related complications ( $31.8 \%$ vs $6.5 \%, \mathrm{p}<0.001)$, the most common of which were chemical meningitis requiring readmission (6 patients) and postoperative headache/emesis requiring readmission (4 patients). Patients in the hydrocephalus group also had significantly longer hospital stays compared with those in the no-hydrocephalus group (mean $4.6 \pm 20.3$ days vs $5.4 \pm 3.5$ days, $\mathrm{p}<0.001$ ).

For our multivariable logistic regression model, we initially included all variables with a $\mathrm{p}<0.05$ in our univariate analysis. Variables we thought might be directly connected to CSF diversion (i.e., wound/non-wound complications, CSF leak, need for fusion, and length of stay) were removed prior to derivation of our final model. On multivariable regression analysis (Table 2), higher intra-
TABLE 1. Univariate analysis for hydrocephalus versus no hydrocephalus groups

\begin{tabular}{|c|c|c|c|}
\hline \multirow[b]{2}{*}{ Variable } & \multicolumn{2}{|c|}{ Hydrocephalus (\%) } & \multirow{2}{*}{$\begin{array}{c}p \\
\text { Value }^{*}\end{array}$} \\
\hline & Yes $(n=22)$ & No $(n=275)$ & \\
\hline Younger age & $13(59.1)$ & $86(31.3)$ & 0.008 \\
\hline Mean LOS & $14.6 \pm 20.3$ & $5.4 \pm 3.5$ & $<0.001$ \\
\hline No. of females & $11(50)$ & $146(53.1)$ & 0.780 \\
\hline Ethnicity & & & 0.354 \\
\hline Caucasian & $16(72.7)$ & $233(84.7)$ & \\
\hline Hispanic/Latino & $5(22.7)$ & $27(9.8)$ & \\
\hline Asian & $0(0)$ & $1(0.4)$ & \\
\hline American Indian & $1(4.5)$ & $4(1.5)$ & \\
\hline African American & $0(0)$ & $0(0)$ & \\
\hline Pacific Islander & $0(0)$ & $2(0.7)$ & \\
\hline Other/unknown & $0(0)$ & $8(2.9)$ & \\
\hline Payer & & & 0.150 \\
\hline Medicaid & $7(31.8)$ & $58(21.1)$ & \\
\hline Private & $15(68.2)$ & $183(66.5)$ & \\
\hline Unknown & $0(0)$ & $34(12.4)$ & \\
\hline No. of prior decompressions & & & 0.001 \\
\hline 0 & $17(77.3)$ & $254(92.4)$ & \\
\hline 1 & $3(13.6)$ & $19(6.9)$ & \\
\hline 2 & $2(9.1)$ & $1(0.4)$ & \\
\hline 3 & $0(0)$ & $1(0.4)$ & \\
\hline Concurrent/postop fusion & $7(31.8)$ & $29(10.5)$ & 0.003 \\
\hline Surgery type & & & 0.062 \\
\hline Duraplasty only & $0(0)$ & $12(4.4)$ & \\
\hline Tonsillar shrinking & $20(90.9)$ & $258(93.8)$ & \\
\hline Tonsillar resection & $2(9.1)$ & $5(1.8)$ & \\
\hline 4th ventricular web & $9(40.9)$ & $37(13.5)$ & 0.001 \\
\hline Postop CSF leak & $5(22.7)$ & $12(4.4)$ & $<0.001$ \\
\hline Wound complication & & & $<0.001$ \\
\hline None & $10(45.5)$ & $243(88.4)$ & \\
\hline Pseudomeningocele & $9(40.9)$ & $24(8.7)$ & \\
\hline Superficial infection & $1(4.5)$ & $8(2.9)$ & \\
\hline Deep infection & $2(9.1)$ & $0(0)$ & \\
\hline Other complications & $7(31.8)$ & $18(6.5)$ & $<0.001$ \\
\hline Mean intraop blood loss (ml) & $126.6 \pm 187.6$ & $56.6 \pm 90.9$ & 0.002 \\
\hline
\end{tabular}

LOS = length of stay.

Mean values are presented \pm SD.

* A p value $<0.05$ was considered statistically significant. Boldface type indicates statistical significance.

operative blood loss (OR 1.003, 95\% CI 1.001-1.006), age $<6$ years old (OR 3.342, 95\% CI 1.282-8.713), and the presence of a fourth ventricular web (OR 3.752, 95\% CI 1.306-10.783) remained significantly associated with the need for post-CM-I decompression CSF diversion.

Of note, when we included all variables with $\mathrm{p}<0.05$ shown in our univariate analysis in our multivariable logistic regression model, fourth ventricular web and intraoperative blood loss remained significantly associated with placement in the hydrocephalus group, although age $<6$ years old did not. 


\section{Illustrative Cases \\ Case 1}

This patient was a 17-year-old girl with a history of severe headaches and progressive myelopathy. Imaging revealed evidence of CM-I in addition to a significant cervicothoracic syrinx. She underwent PFD, duraplasty, and shrinking of the cerebellar tonsils. During surgery, a residual circular sinus was encountered, and approximately $300 \mathrm{ml}$ of blood loss occurred intraoperatively. On exploration of the fourth ventricle, significant arachnoid adhesions were noted over the outlet, and these were dissected. During the procedure, an external ventricular drain was placed, from which the patient was initially weaned without any evidence of elevated intracranial pressures and subsequently removed. Soon afterward, however, the patient began complaining of severe headaches and progressive lethargy. Imaging showed increased ventricular size, and an external ventricular drain was placed emergently, alleviating her symptoms. She subsequently underwent a ventriculoperitoneal shunt placement 9 days after her PFD. By the 3-month follow-up, the patient's syrinx had largely resolved.

\section{Case 2}

This patient was a 4-year-old girl with a history of headaches and sleep apnea. Imaging revealed evidence of CM-I, and the patient subsequently underwent PFD, duraplasty, and shrinking of her cerebellar tonsils. Significant arachnoid adhesions were noted in the region of the tonsils and the fourth ventricular outlet, and the adhesions were dissected. Despite these measures, the patient continued to have progressively worsening symptoms. Imaging demonstrated no evidence of ventriculomegaly, but the patient's tonsillar herniation had persisted. Because her apnea was worsening, she was taken to the operating room for a trial of an external ventricular drain. On placement of the drain, the patient's CSF was noted to be under very high pressure, and the decision was subsequently made to place a ventriculoperitoneal shunt. At last follow-up, the patient's apnea was noted to have improved and stabilized, as had her headaches.

\section{Discussion}

We present, to the best of our knowledge, the first study to examine clinical factors associated with the need for CSF diversion after PFD and duraplasty for CM-I. Patients who are younger, who have greater intraoperative blood loss, and in whom a fourth ventricular web/veil obstructing CSF flow is demonstrated during surgery are significantly more likely to need a subsequent CSF diversion procedure after CM-I decompression. We term this association "Chiari-related hydrocephalus."

In our study, the association between younger age and the need for CSF diversion may be due to the unique characteristics of this patient group. Previous studies ${ }^{1}$ have suggested that these patients present with a significantly higher rate of oropharyngeal dysfunction, syringomyelia, and scoliosis compared with their older peers. The cause of this variation has not been explained, although if abnor-
TABLE 2. Multivariable logistic regression analysis for hydrocephalus versus no hydrocephalus groups

\begin{tabular}{llc}
\hline \multicolumn{1}{c}{ Variable } & \multicolumn{1}{c}{ OR $(95 \% \mathrm{Cl})$} & $\mathrm{p} \mathrm{Value}$ \\
\hline Age $<6$ yrs & $3.342(1.282-8.713)$ & $\mathbf{0 . 0 1 4}$ \\
\hline Intraop blood loss (per ml) & $1.003(1.001-1.006)$ & $\mathbf{0 . 0 1 6}$ \\
\hline 4th ventricular web & $3.752(1.306-10.783)$ & $\mathbf{0 . 0 1 4}$ \\
\hline Previous decompressions & & 0.290 \\
\hline 0 & $1.00($ ref $)$ & $\mathrm{NA}$ \\
\hline 1 & $1.070(0.238-4.819)$ & 0.930 \\
\hline 2 & $13.570(0.963-191.302)$ & 0.053 \\
\hline 3 & $0(0)$ & 1.000 \\
\hline NA = not applicable; ref = reference. & \\
*A p value < 0.05 was considered statistically significant. Boldface type \\
indicates statistical significance.
\end{tabular}

mal CSF hydrodynamics play a role, it is logical that higher rates of hydrocephalus might be seen in these patients.

Higher intraoperative blood loss may lead to higher rates of CRH because of the mechanical and chemical effects of blood products on the CSF circulation system. ${ }^{8}$ Although meticulous attention to hemostasis and clearance of operative blood products prior to dural closure is standard at our institution, it is likely that higher volumes of bleeding during the procedure leads to more blood entering the CSF circulation system and more inflammation and, hence, a higher likelihood of hydrocephalus.

The intraoperative finding of a veil over the fourth ventricular outflow tract is rarely noted in the literature. Its role in the development of post-decompression hydrocephalus is difficult to discern as a result. It is possible that such a veil is suggestive of a baseline deranged CSF flow, and thus patients with the veil are at a higher risk for the development of hydrocephalus and the need for CSF diversion because of this abnormal flow. Indeed, this variable was the most strongly correlated with post-decompression hydrocephalus, with an odds ratio of 3.8. Of the 46 patients in whom a fourth ventricular veil was discovered intraoperatively, $20 \%$ eventually required long-term CSF diversion postoperatively.

Our study has several limitations. The retrospective nature of our investigation naturally makes it vulnerable to confounding. Prospective analysis of this topic is needed, but a prospective study is difficult because of the rarity of post-CM-I decompression hydrocephalus necessitating CSF diversion; we found an incidence of $7.4 \%$, which is comparable to similarly low rates reported in other studies. ${ }^{15}$ In our study we did not examine radiographic risk factors, which likely also play an important role in the prediction of which patients will develop post-decompression hydrocephalus. Future studies evaluating radiographic variables in these patients, preferably in conjunction with clinical variables, would likely offer more robust associations. A minority of cases in our study (14 of 297 or approximately $5 \%$ ) were repeat decompressions in the same patient, and the inclusion of these raises concerns regarding data independence for our analysis. An analysis excluding these cases, however, results in similar findings, with younger age, higher intraoperative blood loss, and the presence of a fourth ventricular veil remaining significant- 
ly associated with the need for long-term CSF diversion. While we used an age cutoff of 6 years of age to dichotomize our population based on our own data and previously published data, ${ }^{1}$ such age-based divisions are likely inherently controversial. We did perform a separate analysis treating age as a continuous variable, and we found, once again, that younger age at the time of surgery remained significantly associated with the need for long-term CSF diversion.

Two of our significant variables-intraoperative blood loss and the presence of a fourth ventricular web-are also subjective and depend on both surgeon recollection and documentation. It is therefore possible that these factors may have been over- or underestimated because of their subjectivity. Finally, our study only examined PFD in which duraplasty was performed. During the study period at our institution, bone-only PFD was performed in 33 patients, of whom 1 patient (3\%) developed CRH. A similar study performed at an institution where a larger proportion of patients undergo bone-only decompression would be beneficial in comparing rates of this complication between the two procedures.

Despite these limitations, we believe that our study may contribute to discussions with patients both preoperatively and immediately postoperatively regarding their risks of developing post-decompression hydrocephalus and may suggest patient populations that should be monitored more closely for this rare but serious complication.

\section{Conclusions}

The development of CRH is an uncommon but real phenomenon following PFD with duraplasty for CM-I. Patients who are younger than 6 years of age, who experience extensive intraoperative blood loss, and who are found during surgery to have a fourth ventricular web obstructing CSF flow may be at higher risk for the development of CRH. These high-risk patients may benefit from careful postoperative follow-up to detect the early signs of hydrocephalus.

\section{References}

1. Albert GW, Menezes AH, Hansen DR, Greenlee JD, Weinstein SL: Chiari malformation Type I in children younger than age 6 years: presentation and surgical outcome. J Neurosurg Pediatr 5:554-561, 2010

2. Alzate JC, Kothbauer KF, Jallo GI, Epstein FJ: Treatment of Chiari I malformation in patients with and without syringomyelia: a consecutive series of 66 cases. Neurosurg Focus 11(1):E3, 2001

3. Arnautovic A, Splavski B, Boop FA, Arnautovic KI: Pediatric and adult Chiari malformation Type I surgical series 1965-2013: a review of demographics, operative treatment, and outcomes. J Neurosurg Pediatr 15:161-177, 2015

4. Bollo RJ, Riva-Cambrin J, Brockmeyer MM, Brockmeyer DL: Complex Chiari malformations in children: an analysis of preoperative risk factors for occipitocervical fusion. J Neurosurg Pediatr 10:134-141, 2012

5. Chotai S, Medhkour A: Surgical outcomes after posterior fossa decompression with and without duraplasty in Chiari malformation-I. Clin Neurol Neurosurg 125:182-188, 2014

6. Fakhri A, Shah MN, Goyal MS: Advanced imaging of Chiari 1 malformations. Neurosurg Clin N Am 26:519-526, 2015

7. Foltz EL, DeFeo DR: Double compartment hydrocephalusa new clinical entity. Neurosurgery 7:551-559, 1980

8. Hill A, Shackelford GD, Volpe JJ: A potential mechanism of pathogenesis for early posthemorrhagic hydrocephalus in the premature newborn. Pediatrics 73:19-21, 1984

9. Kennedy BC, Kelly KM, Phan MQ, Bruce SS, McDowell MM, Anderson RC, et al: Outcomes after suboccipital decompression without dural opening in children with Chiari malformation Type I. J Neurosurg Pediatr 16:150-158, 2015

10. Mazzola CA, Fried AH: Revision surgery for Chiari malformation decompression. Neurosurg Focus 15(3):E3, 2003

11. Milhorat TH, Chou MW, Trinidad EM, Kula RW, Mandell $\mathrm{M}$, Wolpert C, et al: Chiari I malformation redefined: clinical and radiographic findings for 364 symptomatic patients. Neurosurgery 44:1005-1017, 1999

12. Shweikeh F, Sunjaya D, Nuno M, Drazin D, Adamo MA: National trends, complications, and hospital charges in pediatric patients with Chiari malformation type I treated with posterior fossa decompression with and without duraplasty. Pediatr Neurosurg 50:31-37, 2015

13. Tubbs RS, Beckman J, Naftel RP, Chern JJ, Wellons JC III, Rozzelle CJ, et al: Institutional experience with 500 cases of surgically treated pediatric Chiari malformation Type I. J Neurosurg Pediatr 7:248-256, 2011

14. Tubbs RS, McGirt MJ, Oakes WJ: Surgical experience in 130 pediatric patients with Chiari I malformations. J Neurosurg 99:291-296, 2003

15. Zakaria R, Kandasamy J, Khan Y, Jenkinson MD, Hall SR, Brodbelt A, et al: Raised intracranial pressure and hydrocephalus following hindbrain decompression for Chiari I malformation: a case series and review of the literature. $\mathbf{B r} \mathbf{~ J}$ Neurosurg 26:476-481, 2012

\section{Disclosures}

The authors report no conflict of interest concerning the materials or methods used in this study or the findings specified in this paper.

\section{Author Contributions}

Conception and design: Brockmeyer, Guan. Acquisition of data: Guan. Analysis and interpretation of data: Guan, Riva-Cambrin. Drafting the article: all authors. Critically revising the article: Brockmeyer, Riva-Cambrin. Reviewed submitted version of manuscript: all authors. Approved the final version of the manuscript on behalf of all authors: Brockmeyer. Statistical analysis: Guan, Riva-Cambrin.

\section{Correspondence}

Douglas L. Brockmeyer, Department of Neurosurgery, Division of Pediatric Neurosurgery, Primary Children's Medical Center, 100 N Mario Capecchi Dr., Salt Lake City, UT 84113. email: douglas.brockmeyer@hsc.utah.edu. 